\title{
Valoración del estado nutricional de pacientes mayores de 65 años en tratamiento sustitutivo en una unidad de diálisis
}

\author{
J uana Gómez Macías
}

Unidad de Diálisis

Fundación J iménez Díaz

Madrid

\section{RESUMEN}

Los ancianos que reciben tratamiento de hemodiálisis crónica han aumentado en la actualidad y esta población es especialmente susceptible a varios problemas, entre ellos la malnutrición, que en hemodiálisis se asocia a una mayor morbimortalidad. La evaluación del estado de nutricional es especialmente importante para las enfermeras de diálisis.

O bjetivo y Ámbito: Conocer el estado de salud y la situación metabólico-nutricional, basados en parámetros antropométricos, de los pacientes mayores de 65 años incluidos en tratamiento de hemodiálisis crónica en una Unidad de Diálisis, con especial referencia al riesgo de padecer desnutrición y conocer el grado de intervención actual sobre los pacientes desnutridos, indicando qué pacientes tienen un aporte suplementario.

Diseño: Se trató de un estudio descriptivo transversal.

Pacientes, material y métodos: Se estudiaron 22 pacientes (16 mujeres y 6 hombres), mayores de 65 años que recibían tratamiento de hemodiálisis crónica en el primer trimestre de 2004. El estado nutricional se evaluó con medidas antropométricas, marcadores bioquímicos, marcadores dialíticos, test MNA de evaluación nutricional y encuestas dietéticas. Las medidas antropométricas fueron realizadas

Correspondencia:
J uana Gómez Macías
Unidad de Diálisis
Fundación J iménez Díaz
Av. Reyes Católicos, 2
28040 Madrid
anagomezmacias@telefónica.net

por personal entrenado y las medidas bioquímicas coincidieron con las extracciones mensuales de la unidad. Se clasificó el grado de desnutrición según los parámetros antropométricos PTC y CB.

Resultados: Según el PTC, el 90,8\% $(n=20)$ de los pacientes padecían de desnutrición calórica (5 leve, 12 moderada, 3 grave) y según el CB, el $54,5 \%(n=12)$ de los pacientes tuvieron malnutrición proteica (7 leve, 5 grave). Los diabéticos presentaron mayor prevalencia de desnutrición calórica con un 66,66\% ( $n=6)$. Un 54,5\% (n=12) de los pacientes recibieron algún suplemento nutricional.

Conclusiones: La malnutrición más frecuente en los pacientes estudiados es la calórica más que la proteica, siendo conveniente utilizar varios criterios (antropométrico, bioquímico o inmunológico) para establecer el diagnóstico de una desnutrición, siendo un gran aliado los parámetros antropométricos y el tratamiento con suplementos nutricionales en la práctica habitual para mantener el estado nutricional y minimizar la morbimortalidad en los pacientes desnutridos.

\section{PALABRAS CLAVE: ANCIANOS \\ HEMODIÁLISIS \\ SITUACIÓN METABÓLICO-NUTRICIONAL}

\begin{abstract}
The elderly population who received hemodialysis treatment has increased in the actually and this population is especially susceptible to have several problems like malnutrition who is associated with higher morbid-mortality in hemodialysis treatment. The evaluation of the nutritional state is especially important for dialysis nurses.
\end{abstract}


O bjective and Scope: To know the state of health and the metabolic-nutritional situation with antropometric parameters of the elderly patients those more than 65 years old treated with chronic hemodialysis in a Dialysis Unit, specially whose had the risk of malnutrition, and to know what was the grade of interventions to patients with malnutrition in the actually and what was the number of patients with nutritional supplements.

\section{Design: Transversal descriptive study.}

Patients, materials and method: The study involved 22 patients (16 female and 6 males), over the age of 65 years old who received chronic hemodialysis treatment in the first three months of 2004. The nutritional state was evaluated in patients over the age of 65 years old who had chronic hemodialysis treatment in the first three months of 2004. Nutritional state was assessed with antropometric measurements, biochemical scores, dialysis scores, test MNA of nutritional assessment and dietary counselling. The antropometric measurements was carried out by person trained in nutritional assessment and boichemical scores was the mensual blood extracs of the Dialysis unit. Malnutrition was classified to antropometric measurements: PTC and CB.

Results: According to the PTC, $90.8 \%(n=20)$ patients had energy malnutrition (5 slight, 12 moderate, 3 severe) and according to $C B, 54.5 \%(n=12)$ patients had protein malnutrition (7 slight, 5 severe). Diabetic patients had more prevalence of energy malnutrition with $66.66 \%(n=6)$ patients. $54.5 \%(n=12)$ patients were taken some nutritional supplements.

Conclusions: Energy Malnutrition is more frequent than Energy Malnutrition in the studied patients and some options is convenient (antropometric, biochemical or inmunological parameters) to diagnose Malnutrition, and antropometric measurements are helpful and the treatment with nutritional supplements is very commonly to keep an optimal Nutritional state and a minimal morbic-mortality in patients who have Malnutrition.

KEYWORDS: $\quad$ ELDERLY PATIENT

HAEMODIALYSIS

THE METABOLIC-NUTRITIONAL SITUATION

\section{INTRODUCCIÓN}

En los últimos años, se ha desarrollado un interés creciente hacia los aspectos nutricionales de los pacientes ancianos tratados con hemodiálisis $(H D)$, su número se ha incrementado, estimándose que los ancianos en tratamiento renal sustitutivo son el 39\% del total de pacientes ${ }^{1}$. Los ancianos presentan una se- rie de problemas generales, sumándose otros asociados a la HD y a la insuficiencia renal crónica (IRC), que influyen en la valoración de su inclusión en programa de tratamiento sustitutivo y que además condicionarán el resultado $2,3,4,5$, se incrementa el riesgo de desnutrición con respecto a pacientes jóvenes $^{6}$. Varios trabajos publicados, demuestran un porcentaje alto de pacientes con rasgos de desnutrición en los que su aparición se asocia a una mayor morbimortalidad ${ }^{7,8,9}$. La nutrición se considera como un marcador pronóstico fundamenta ${ }^{10}$. La población anciana con desnutrición, presenta un deterioro progresivo en su estado general, un aumento de la susceptibilidad ante las agresiones y más predisposición a la aparición de más enfermedades y problemas, lo que se traduciría en una peor calidad de vida ${ }^{11}$. Es muy importante definir la situación nutricional12,13,14 considerando la desnutrición como una enfermedad con entidad propia, que requiere un abordaje específico para su diagnóstico y tratamiento. No hay un parámetro sencillo y reproducible para definirla con precisión, por lo que su diagnóstico es complejo y basado en métodos bioquímicos, la cinética de la urea, antropometría y encuesta dietética ${ }^{15}$.

\section{OBJETIVOS}

1. Conocer el estado de salud y la situación metabólico-nutricional, basados en parámetros antropométricos, de los pacientes mayores de 65 años incluidos en tratamiento crónico de hemodiálisis, con especial referencia al riesgo de padecer desnutrición.

2. Conocer el grado de intervención actual sobre los pacientes desnutridos, indicando qué pacientes tienen un aporte suplementario.

\section{PACIENTES, MATERIAL Y MÉTODOS}

Se trató de un estudio descriptivo transversal que recogió a todos los pacientes mayores de 65 años que recibían tratamiento de HD crónica en el primer trimestre de 2004 en una Unidad de Diálisis. Tras informarles de la metodología del estudio, dieron su consentimiento. Se seleccionaron a 22 pacientes, 16 mujeres y 6 hombres, con mediana $75(5,92)$ años de edad, que llevaban en tratamiento sustitutivo una media de $20,72(19,65)$ meses. Recibieron tratamiento con HD convencional con bicarbonato y duración media de la sesión de diálisis de 223,63 $(24,01)$ minutos. El flujo de sangre fue de 300 a $350 \mathrm{ml} / \mathrm{min}$ y el flujo baño de HD de 500 a 800 $\mathrm{ml} / \mathrm{min}$.

Los datos recogidos fueron los siguientes:

1. Datos generales: edad, sexo, patología de base, índice de comorbilidad de Charlson, tiempo en HD, integridad dentaria, tratamiento farmacológico: quelantes de fósforo, do- 
sis de EPO, suplementos nutricionales (vía oral y/ o nutrición parenteral intradiálisis) y presencia de diabetes mellitus.

2. Parámetros bioquímicos: hematocrito, hemoglobina, leucocitos, linfocitos, ferritina, transferrina, índice de saturación de hierro (\%), bicarbonato, PTH, proteína C reactiva, creatinina, NUS, fósforo, albúmina, proteínas totales, prealbúmina y colesterol.

3. Parámetros de valoración nutricional. En el registro antropométrico: talla, circunferencia del brazo (CB), pliegue tricipital (PCT), índice de masa corporal 0 índice de Q uetelet (IMC), circunferencia muscular del brazo (CMB) y área muscular del brazo (AMB). Para evaluar el estado nutricional se utilizó el test MNA (Mini Nutritional Assessment) ${ }^{16}$ y encuestas alimentarias de tres días.

4. Parámetros dialíticos: peso seco post HD ( $\mathrm{kg})$, tiempo de diálisis (min), acceso vascular, tipo de membrana, $\mathrm{Kt} / \mathrm{V}$ calculada mediante fórmula de Gotch y tasa de catabolismo proteico calculada mediante el PCR ( $/ \mathrm{kg} /$ día) para estimar la ingesta proteica.

Todos los parámetros bioquímicos correspondieron al programa de extracción de analítica establecido en la Unidad de Diálisis, con el fin de no someter a extracciones de sangre adicionales a los pacientes.

Los tratamientos farmacológicos se recogieron de los datos de las historias clínicas. Los suplementos nutricionales que los pacientes tomaron fueron: Nepro ${ }^{\circledR}$ (Abbott Lab), Renapro ${ }^{\circledR}$ (Bionet Med.) y la nutrición parenteral administrada intradiálisis, constituida en el Departamento de Farmacia del hospital. La medición de los parámetros antropométricos se realizó al término de la sesión de HD de mitad de semana, siguiendo las normas de Alastrué17. Los instrumentos de medida fueron una cinta métrica inextensible, un calibrador de panículo adiposo tipo Harpender (0-2 mm) J ohn Bull Caliper Skinfold ${ }^{\circledast}$ y una báscula con tallímetro. Las mediciones fueron realizadas por un sólo observador previamente entrenado y siguiendo las recomendaciones de la DOQI ${ }^{18}$. La valoración de la composición corporal se realizó por métodos antropométricos. Los valores obtenidos del PTC y CB se expresaron con arreglo a su relación porcentual con los valores del Pth (percentil) 50 de una población sana de referencia del mismo grupo de edad y sexo descrita por Alastrué, que según su grado, sirvió para valorar el estado de desnutrición en tres niveles. Para determinar la malnutrición energético-calórica, se consideró el PTC, el grado de desnutrición proteica y la CB. La complexión corporal se estimó según el IMC y se siguió la clasificación de la OMS de 1995. El test MNA, se calificó mayor de 23,5 normal, de 17 a 23,5 puntos riesgo de malnutrición y menos de 17 puntos malnutrición. Se realizaron encuestas alimentarias, recordatorios de 3 días y de entrevistas del observador con cada paciente, para confirmar y ajustar los datos del recordatorio. No se hizo una cuantificación de los nu- trientes de la encuesta, pero se aplicó para el desarrollo y cumplimentación del test MNA. El Kt/ V y PCR ( $/ \mathrm{kg} / \mathrm{día}$ ) se obtuvieron mediante programa informático usando la urea pre y post HD de la sesión de mitad de semana. Las muestras post- HD fueron extraídas a los cinco minutos de finalizar la sesión de diálisis.

Para el análisis estadístico, la comparación entre variables continuas y cuantitativas se realizó mediante análisis de la varianza (ANOVA) y mediante prueba t de Student. El test de Bonferroni se usó para las comparaciones múltiples Post-hoc. La comparación de las diferentes variables cualitativas se realizó mediante Chi-cuadrado. La hipótesis nula se rechazó en el análisis estadístico si era $p<0.05$. El análisis estadístico se realizó con el paquete informático SPSS 10.00 para Windows.

\section{RESULTADOS}

Las diferentes etiologías de base de la IRC de los pacientes del estudio se recogen en la figura 1.

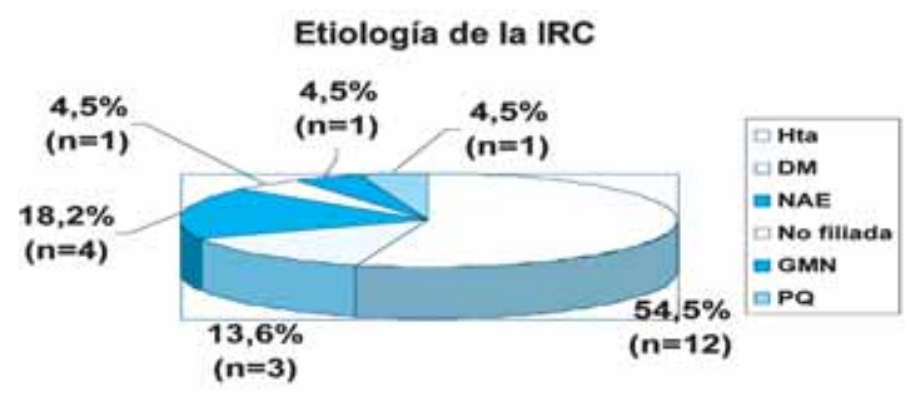

Figura 1. Etiologías de base de la IRC de los pacientes.

El estudio de su integridad dentaria demostró que al 18,2\% $(n=4)$ les faltaban algunas piezas dentales y el resto, $81,8 \%$ $(n=18)$ tenían íntegra la dentadura (postiza o natural).

Los pacientes presentaron un índice de comorbilidad de Charlson media $7,45(2,04)$ y mediana 7,5 .

Aunque los pacientes, además de la etiología de la nefropatía, presentan otras patologías, destaca la Diabetes Mellitus tipo-II (siendo etiología y también enfermedad intercurrente e incluida en el índice de comorbilidad de Charlson), estando presente en un $40,9 \%(n=9)$ de la totalidad de los pacientes.

Los valores media (DE) de los parámetros bioquímicos aparecen recogidos en la tabla 1 . Los resultados obtenidos por sexos en cuanto a parámetros antropométricos se recogen en la tabla 2. Se separaron varios grupos como desnutrición calórica y desnutrición proteica que se recoge en la figura 2. 


\begin{tabular}{l|l}
\hline Parámetros & Media (DE) \\
\hline Hemoglobina (gr/ dl) & $10,95(1,10)$ \\
\hline Linfocitos (\%) & $17,23(5,37)$ \\
\hline Transferían (mg/ dl) & $178,95(29,11)$ \\
\hline Índice de saturación de hierro (\%) & $19,89(5,64)$ \\
\hline Ferritina (ng/ ml) & $370,31(281,27)$ \\
\hline NUS (mg/ dl) & $73,32(12,31)$ \\
\hline Creatinina (mg/ dl) & $8,26(2,02)$ \\
\hline PTH (pg/ ml) & $270,71(163,07)$ \\
\hline Fósforo (mg/ dl) & $5,05(1,16)$ \\
\hline Bicarbonato (mEq/ L) & $22,95(2,31)$ \\
\hline Kt/ V & $1,26(14,75)$ \\
\hline n PCR (g/ kg/ día) & $0,88(0,15)$ \\
\hline Proteínas tot (gr/ dl) & $6,45(0,53)$ \\
\hline Albúmina (mg/ dl) & $3,51(035)$ \\
\hline Prealbúmina (mg/ dl) & $24,94(7,48)$ \\
\hline Colesterol (mg/ dl) & $167,36(40,13)$ \\
\hline Proteína C reactiva (mg/ dl) & $2,07(3,25)$ \\
\hline
\end{tabular}

Tabla 1. Parámetros bioquímicos.

\begin{tabular}{lcc}
$\begin{array}{l}\text { Valores } \\
\text { Antropométricos }\end{array}$ & Hombres. Media (DE) & Mujeres. Media (DE) \\
$\begin{array}{l}\text { Talla }(\mathbf{c m}) \\
\text { Peso seco (Kg) }\end{array}$ & $168,33(8,63)$ & $150,29(6,90)$ \\
\hline PTC (mm) & $13,16(11,25)$ & $60,15(15,61)$ \\
CB $(\mathbf{c m})$ & $28,13(3,18)$ & $14,28(6,36)$ \\
IMC (Kg/ m ${ }^{2)}$ & $24,21(4,79)$ & $26,53(5,51)$ \\
\hline
\end{tabular}

Tabla 2. Parámetros antropométricos.

\section{Grupos con Desnutrición (\%)}

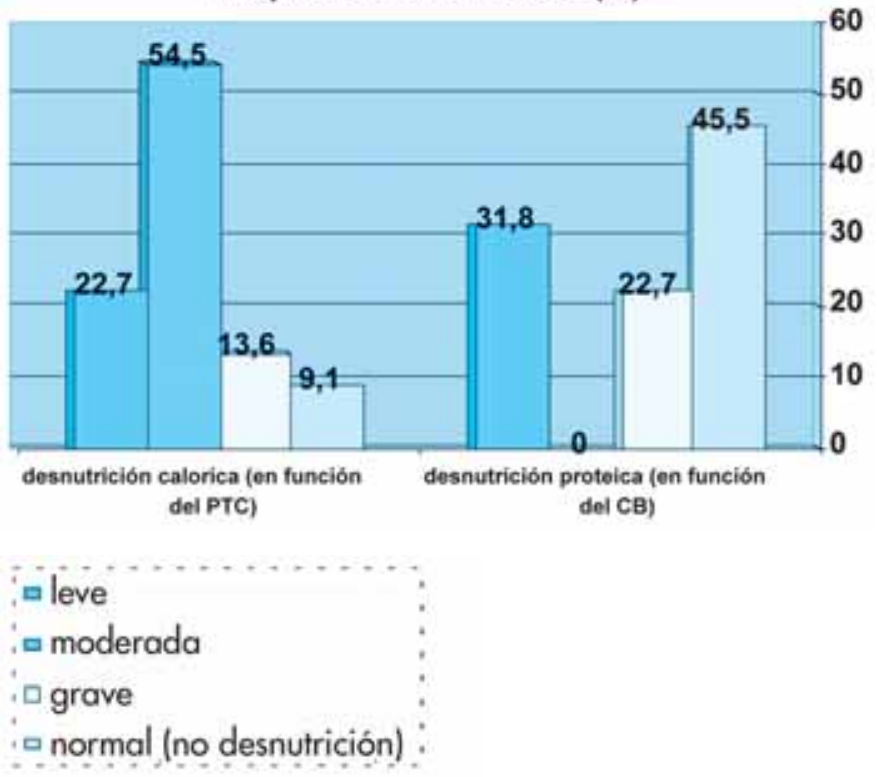

Figura 2. Grados de desnutrición calórica y proteica.
Se estudiaron separadamente la situación nutricional de los diabéticos $(40,9 \%)$ y los resultados se reflejan en la tabla 3. En cuanto al test MNA de valoración nutricional, se encontraron que un $31,8 \%(n=7)$ estaban clasificados como normales, había $36,4 \%(n=8)$ en riesgo de desnutrición y con malnutrición, un $31,4 \%(n=7)$.

\begin{tabular}{|c|c|c|c|c|}
\hline $\begin{array}{l}\text { Desnutrición } \\
\text { Calórica }\end{array}$ & $\begin{array}{c}\text { Leve } \\
33,33 \% \\
(n=3)\end{array}$ & $\begin{array}{c}\text { Moderada } \\
22,22 \% \\
(n=2)\end{array}$ & $\begin{array}{c}\text { Grave } \\
11,11 \% \\
(n=1)\end{array}$ & $\begin{array}{c}\text { Normal } \\
33,33 \% \\
(n=3)\end{array}$ \\
\hline $\begin{array}{l}\text { Desnutrición } \\
\text { Proteica }\end{array}$ & & & $\begin{array}{c}22,2 \% \\
(n=2)\end{array}$ & $\begin{array}{c}77,8 \% \\
(n=3)\end{array}$ \\
\hline
\end{tabular}

Tabla 3. Situación nutricional de los pacientes diabéticos.

Se quiso conocer el grado de intervención sobre la desnutrición en los ancianos y se vio qué características tenían a los que se administró algún suplemento nutricional (oral o parenteral). El grupo recibía aportes suplementarios nutricionales en un total de $54,5 \%(n=12)$ pacientes, con $9,1 \%(n=2)$ fueron NPID, un $36,4 \%(n=8)$ suplementos orales y un $9,1 \%(n=2)$ recibieron ambos suplementos.

En cuanto a otros tratamientos, un $59,1 \%(n=13)$ de los pacientes tomaron carbonato cálcico (ligante de fósforo) y la dosis media de rHu-EPO fueron 9909,09 $(6975,52)$ unidades/ semana.

Los accesos vasculares para hemodiálisis fueron: catéteres permanentes $36,4 \%(n=8)$, fístula nativa $36,4 \%(n=8)$ y fístula protésica $27,3 \%(n=6)$.

En el $54,5 \%(n=12)$ se usaron membranas sintéticas y en el $45,5 \%(n=10)$ membranas de diacetato o triacetato de celulosa.

La desnutrición calórica estuvo presente en $50 \%(n=3)$ de los pacientes varones frente al $75 \%(n=12)$ de las mujeres. Respecto a la desnutrición proteica, ésta estuvo presente en un $31,3 \%(n=5)$ de las pacientes mujeres y no dándose ningún caso en los varones. En el caso de la desnutrición calórica, los pacientes que se dializaron con membranas sintéticas fueron un $20 \%(n=2)$ frente el $80 \%(n=8)$ que se dializaron con membranas celulósicas. $Y$ en la desnutrición proteica, un $16,7 \%$ $(n=2)$ fueron membranas sintéticas frente el $30 \%(n=3)$ de las membranas celulósicas.

Se estudiaron con el test ANOVA, las características de los distintos grupos con desnutrición calórica en función del PTC (normales, leve, moderada, grave), se encontraron diferencias estadísticamente significativas en parámetros antropométri$\cos , C M B, A M B$ y en la dosis de carbonato cálcico que recibieron, como se puede ver en la tabla 4. 


\begin{tabular}{|l|c|c|c|c|c|c|c|}
\hline & $\begin{array}{c}\text { Normo- } \\
\text { calóricos } \\
\text { Media (DE) }\end{array}$ & $\begin{array}{c}\text { Desnutrición } \\
\text { calórica leve } \\
\text { Media (DE) }\end{array}$ & $\begin{array}{c}\text { Desnutrición } \\
\text { calórica } \\
\text { moderada } \\
\text { Media (DE) }\end{array}$ & $\begin{array}{c}\text { Desnutrición } \\
\text { calórica } \\
\text { grave } \\
\text { Media (DE) }\end{array}$ & p & F & gl \\
\hline $\begin{array}{l}\text { Carb } \\
\text { (gr) }\end{array}$ & $0(0)$ & $0,83(0,28)^{*}$ & $4,21(1,97)^{*}$ & $12,66(8,3)^{*}$ & 0,039 & 4,26 & 18 \\
\hline $\begin{array}{l}\text { CMB } \\
\text { (cm) }\end{array}$ & $68,27(13,59)$ & $15,49(4,23)^{*}$ & $26,33(13,59)^{*}$ & $49,23(20,45)^{*}$ & 0,01 & 8 & 18 \\
\hline $\begin{array}{l}\text { AMB } \\
\left(\mathbf{c m}^{2}\right)\end{array}$ & $2384,74(1918,3)$ & $9,08(12,23)^{*}$ & $155,37(278,52)^{*}$ & $1128,4(1584,17)^{*}$ & 0,015 & 4,59 & 18 \\
\hline
\end{tabular}

Carb: los que toman carbonato cálcico; CMB: circunferencia media del brazo; AMB: área muscular del brazo; $p$ : valor de la p; F:valor de la F; gl: grado de libertad;* $p<0.05$ entre grupo normal y leve, entre grupo normal y moderado, y entre grupo normal y grave.

Tabla 4. Características de los distintos grupos con desnutrición calórica en función del PTC.

Se estudiaron con el test ANOVA los distintos grupos con desnutrición proteica en función del CB (normales, leve, moderada, grave), no se encontraron diferencias estadísticamente significativas en ninguno de los parámetros estudiados excepto con peso seco, linfocitos, proteínas totales y transferrina. Las características de los grupos según el grado de desnutrición proteica, se reflejan en la tabla 5.

\begin{tabular}{|l|c|c|c|c|c|c|c|}
\hline & $\begin{array}{c}\text { Normo- } \\
\text { protéicos } \\
\text { Media(DE) }\end{array}$ & $\begin{array}{c}\text { Desnutrición } \\
\text { proteíca leve } \\
\text { Media(DE) }\end{array}$ & $\begin{array}{c}\text { Desnutrición } \\
\text { Protéica } \\
\text { Moderada }\end{array}$ & $\begin{array}{c}\text { Desnutrición } \\
\text { protéica } \\
\text { grave } \\
\text { Media(DE) }\end{array}$ & p & F & gl \\
\hline $\begin{array}{l}\text { PS } \\
\text { (kg) }\end{array}$ & $73,48(13,65)$ & $54,21(5,97)^{*}$ & 0 & $51,4(9,71)^{*}$ & 0.031 & 0.83 & 19 \\
\hline $\begin{array}{l}\text { Pr T } \\
\text { (g/dl) }\end{array}$ & $6,45(0,53)$ & $6,79(0,57)^{*}$ & 0 & $6(0,34)^{*}$ & 0.030 & 4.22 & 19 \\
\hline $\begin{array}{l}\text { Trasf } \\
\text { (mg/dl) }\end{array}$ & $184,2(26,39)$ & $190,85(27,02)^{*}$ & 0 & $151,8(23,43)^{*}$ & 0.045 & 3.66 & 19 \\
\hline Linf (\%) & $16,39(5,23))$ & $19,73(5,52)^{*}$ & 0 & $17,45(5,93)^{*}$ & 0.019 & 1.19 & 19 \\
\hline
\end{tabular}

PS: peso seco; Prot tot: proteínas totales; Trasf: transferrina; Linf: linfocitos; p: valores de la p; F: valores de la F; gl: grado de libertad. ${ }^{*} p<0.05$ entre grupo normal y leve, y entre grupo normal y grave.

Tabla 5. Características de los distintos grupos con desnutrición proteica en función del CB

\section{DISCUSIÓN}

Este estudio describe la situación nutricional de un grupo de ancianos tratados con hemodiálisis. Los datos indican que existe una elevada prevalencia de desnutrición, sobre todo con un mayor porcentaje de pacientes con desnutrición calórica. Un porcentaje elevado de ancianos dializados son los diabéticos independientemente de la causa de IRC y en este grupo también es frecuente la desnutrición calórica. No se encontraron diferencias en aquellos parámetros que podían haber determinado la situación nutricional como: la integridad dentaria o la utilización de una determinada membrana. Los pacientes también presentaron valores de adecuación de diálisis y parámetros bioquímicos dentro de los recomendados por la guía DOQ ${ }^{18}$.
La desnutrición calórico-proteica es frecuente en los pacientes en hemodiálisis. Varía la prevalencia según los diversos autores, dependiendo de los métodos de valoración utilizados ${ }^{19}$. En el análisis de este estudio, basado en parámetros antropométricos ${ }^{20}$, se encontró que los pacientes presentan desnutrición tanto calórica como proteica, con un porcentaje más elevado de malnutrición calórica, al contrario de lo que aparece en muchos de los estudios, aunque en las encuestas dietéticas sí muestran que en la ingesta predomina el déficit calórico.

Los pacientes que están con desnutrición calórica pueden presentar un deterioro general, con disminución del peso corporal, apatía, debilidad general, etc, sin tener cambios en los parámetros bioquímicos. En este estudio no se pudo correlacionar cambios significativos a nivel de parámetros bioquímicos en los que presentaron desnutrición calórica. En la desnutrición proteica sí se pueden apreciar cambios bioquímicos con una depleción de las proteínas séricas con descenso en los niveles de albúmina, prealbúmina y transferrina, más afectación en el sistema inmunocompetente, con un descenso en los niveles de linfocitos. En este estudio se vio que los pacientes con desnutrición proteica grave, presentaron una media de transferrina $151,8(23,43) \mathrm{mg} / \mathrm{dl}$, unas proteínas totales de $6(0,34) \mathrm{g} / \mathrm{dl}$, un descenso en los linfocitos con una media $17,45(5,93)$ y una bajada el peso seco con $51,4(9,71) \mathrm{kg}$. La hipoalbuminemia se asocia a una mayor morbimortalidad y a un origen multifacto rial, pudiendo estar relacionado con factores no nutricionales como son la inflamación y situaciones comórbidas ${ }^{11,19}$. En este estudio, la mayoría de los pacientes presentaron niveles de albúmina menores a 3,6 gr/ dl, incluso los pacientes que estaban normonutridos desde el punto de vista antropométrico. La albúmina fue un marcador que no se asoció significativamente con otros. En los parámetros bioquímicos, se pudo apreciar que la albúmina, prealbúmina y transferrina se encontraban por debajo de lo recomendado por la guía de nutrición de la NFK-DOQ ${ }^{18}$.

En los diabéticos también encontramos mayor prevalencia de desnutrición calórica que proteica. Los resultados mostrados en el presente estudio, son similares al grupo de trabajo de Manzano y $\mathrm{col}^{14}$, presentando los pacientes diabéticos más desnutrición calórica que proteica.

En cuanto a las causas que provocaron la desnutrición, no se vio significación en la falta de piezas dentales, que en estos pacientes no provocó desnutrición, resultado similar a otro grupo de trabajo ${ }^{21}$. En cuanto al tipo de membranas utilizadas en este trabajo no se pudo objetivar una relación significativa si influían en la aparición de la desnutrición.

Los pacientes del estudio presentaron un $\mathrm{Kt} / \mathrm{V}$ de 1,26 $(14,75)$, dentro del rango recomendado ${ }^{18}$, pero estaban por debajo con respecto al nPCR 22, siendo de $0,88(0,15)$ g/ kg/ día. 
En cuanto a la complexión corporal se vio que las mujeres presentaron un IMC de $26,14(6,8)$ tendentes al sobrepeso, y los hombres con un IMC 24,21 $(4,7)$ tendiendo a normal. En una revisión de enfermos en $\mathrm{HD}$, encontraron que el sobrepeso es la causa principal alteración nutricional en esta población, apareciendo en el $38 \%$ de los enfermos y que es más frecuente en mujeres y en diabéticos tipo 2, aumentando también con la edad ${ }^{23}$. En cuanto al test MNA de valoración nutricional, al compararlo con los resultados obtenidos con PTC Y CB, no se encontró relación estadística entre ellos, nos puede ayudar a la hora de evaluar a los pacientes como otro método complementario.

En los pacientes de hemodiálisis en los que se incluyen soportes nutricionales orales, ante la desnutrición ya instaurada, la NPID, representa el siguiente paso para los que no toleraron 0 no se trataron con éxito con suplementos enterales. En el presente estudio, los pacientes recibieron aportes suplementarios nutricionales en un total de $54,5 \%(n=12)$ pacientes de los que el $9,1 \%$ ( $n=2$ ) fueron NPID (vía parenteral intradiálisis), un $36,4 \%(n=8)$ suplementos orales y un $9,1 \%(n=2)$ pacientes recibieron ambos suplementos, siendo habitual la prescripción de los suplementos orales.

Se utilizaron varios métodos para definir la situación nutricional de los pacientes, parámetros bioquímicos, antropométricos y test MNA. Se eligió para estudiarlos los parámetros antropométricos porque este es el método avalado por la literatura ${ }^{12,14,24,11,20}$. Esto nos permitió ver, cómo usando sólo parámetros bioquímicos, que es el parámetro por el que se define habitualmente la desnutrición en los pacientes, existe un amplio grupo de pacientes que presentan desnutrición calórica, e incluso proteica sin que exista repercusión bioquímica. De hecho, sólo los pacientes con desnutrición proteica severa, presentaban una concentración más baja de proteínas y transferrina. Esto apunta hacia la necesidad de un cambio en la práctica asistencial para determinar con más fiabilidad qué pacientes presentan algún grado de desnutrición y comenzar antes un soporte nutricional adecuado.

El haber realizado un corte transversal, por lo que pueden existir factores no tenidos en cuenta y ser un estudio con pocos casos son limitaciones que deben tenerse en cuenta. Como ventajas se trata de un grupo homogéneo, tratado por un mismo grupo de profesionales, lo que ayuda a definir la forma de trabajar y la manera de cambiar o ajustar mejor las necesidades.

\section{CONCLUSIONES}

1. La malnutrición más frecuente en los pacientes estudiados es la calórica más que la proteica, probablemente porque aparezca antes.
2. Es conveniente utilizar varios criterios (antropométrico, bioquímico o inmunológico) para establecer el diagnóstico de una desnutrición ya que nos aportan en conjunto una valiosa información sobre el estado nutricional de una persona.

3. La albúmina y el resto de los parámetros bioquímicos se alteran tardíamente en situaciones graves, por lo que es necesario usar parámetros más sensibles que determinen antes la desnutrición, teniendo un gran aliado en los parámetros antropométricos.

4. Uno de los tratamientos más recomendados para los ancianos en riesgo de desnutrición o los desnutridos en hemodiálisis, es el tratamiento con suplementos para mantener el estado nutricional y minimizar la morbimortalidad.

5. La valoración continuada del estado nutricional constituye el primer paso en el tratamiento de cualquier estado de desnutrición.

\section{AGRADECIMIENTOS}

Quisiera agradecer para la realización de este trabajo, la colaboración de los pacientes de la unidad, de mis compañeras y en especial, a la Dra. Albalate por la ayuda prestada y su disponibilidad en todo momento.

\section{BIBLIOGRAFÍA}

1. Ortega $F$, Aspectos éticos y sociales del tratamiento dialítico en Nefrología Clínica. Hernando L, Ed. Médica Panamericana SA 2003; 19:813-17.

2. Martín Salinas $C$, Martínez Montero $P$, Alimentación en la edad avanzada en Nutrición y dietética. Martín Salinas C, Ed. Difusión Avances de Enfermería (DAE) 2002; 18:281-295.

3. Guillé $A$, Ronda MJ, Nutrición en la insuficiencia renal en Cuidados de enfermería en la insuficiencia renal. Andrés A, Fortuni C, Ed. ELA 1994; 17:261-285.

4. García-Lorda P, Foz M, Salas-Salvador J , Estado nutricional de la población anciana de Cataluña. Med Clin (Barc) 2002; 118:707-15.

5. De Torres Aured M.L, Malnutrición en Nutrición y dietética. Martín Salinas C, Ed. Difusión Avances de Enfermería (DAE) 2002; 20:321-331.

6. Burrowes JD, Cross-sectional relationship between dietary protein and energy intake, nutritional status, and comorbidity older versus younger hemodialysis patients. [En línea] [Consulta: 16/12/03].

7. Caravaca F, Arrobas M, Pizzarro JL et al, Predictors of early death during dialysis. Nefrología 2001; 21:274-82.

8. Wolfson M, Nutrition in Elderly Dialysis Patients Seminars in Dialysis 2002; 15:113-115.

9. Kopple JD, Effect of nutrition on morbidity and mortality in maintenance dialysis patients. Am J Kidney Dis 1994; 24:1002-1009. 
10. Realch $D$, Match $D E$, Metabolismo proteico y nutrición en Insuficiencia Renal Crónica. Diálisis y Trasplante. Ed. Norma 1997; 23:671-94.

11. Lorenzo $\mathrm{V}$, Rufino $M$, Aspectos nutricionales en hemodiálisis, Valderrábano F, Ed J ims 1999; 24:339-360.

12. Picó Mira L. Valoración del estado nutricional en una unidad de hemodiálisis. Revista de la Sociedad Española de Enfermería Nefrológica 2002; 19:18-22.

13. Guerrero Ricos M.A, Dieta en diálisis peritoneal: malnutrición y alteraciones metabólicas en Diálisis peritoneal. 3a curso andaluz de diálisis peritoneal para enfermería. Espejo L. Ed. SEDEN. Córdoba 2003; 10:127-157.

14. Manzano J.M, Nieto M.D, Valoración nutricional de enfermería de los pacientes tratados con HD en un centro periférico. Libro comunicac. del XXVIII congreso nacional SEDEN. P. Mallorca 2003.

15. Marcen $\mathrm{R}$, Manifestaciones clínicas y bioquímicas de la IRC. En N efrología Clínica. HernandoL, Aljama P, Ed Médica Panamericana 2003; 18:707-711.

16. Asociación de farmacéuticos rurales e Cataluña. Protocolo de evaluación del estado nutricional Mini Nutritional As- sessment (MNA). [En línea]. [Consulta: 28 febrero 2004]. Disponible en : http:/ / www.correofarmaceutico.com

17. Alastrué Vidal A, Sitges Serna A et al, Valoración de los parámetros en nuestra población. Med Clínica 1982; 80:407-415.

18. Guía K/DOQI nutrition in Renal Failure. Am J Kid Dis 2000; 35:51-140.

19. Fernández Reyes MJ, Alvarez Ude F, Estado nutricional, comorbilidad e inflamación en hemodiálisis. Nefrología 2000;20:85-91

20. Millán $D$ et col, Valoración del estado nutricional por parámetros antropométricos en dos unidades de diálisis. Libro de comunicaciones del XXI Congreso de la SEDEN. Salamanca 1996:153-258.

21. Kalantar-Zadeh K, Kopple J D, Nutritional management of patients undergoing maintenance hemodialysis en Nutritional management of renal disease, Kopple JD, Massry SG, Ed Lippincott Williams and Wilkins 2003;25:433-466.

22. Maduell F, Navarro V, Medida y control de la eficacia: La hemodiálisis adecuada. Monitorización contínua en Tratado de HD. Valderrábano F, Ed Médica J ims 1999; 141-149. 